\title{
The Application of Pulsed Radiofrequency to Dorsal Root Ganglion Ameliorates Neuropathic Pain by Reducing CCL2 Expression in the Early Stage After Sciatic Nerve Injury in Rats
}

\section{Cheng-Fu wan}

First Hospital of China Medical University

\section{Bo-Han zhang}

First Hospital of China Medical University

\section{Dao-Song Dong}

First Hospital of China Medical University

Tao Song ( $\nabla$ songtaocmu@163.com )

First Hospital of China Medical University

\section{Research Article}

Keywords: Pulsed Radiofrequency Treatment, Chemokine CCL2, Neuralgia, Sciatic nerve.

Posted Date: December 10th, 2020

DOI: https://doi.org/10.21203/rs.3.rs-119934/v1

License: (c) (1) This work is licensed under a Creative Commons Attribution 4.0 International License.

Read Full License 


\section{Abstract}

Background: Neuropathic pain (NP) can be treated effectively using pulsed radiofrequency (PRF). NP development and maintenance involves the essential neurotransmitter chemokine c-c motif ligand 2 (CCL2). The present study aimed to determine whether PRF regulated CCL2 expression in sciatic nerve injury (SNI) model rats.

Methods: Sprague-Dawley rats were divided randomly into a sham group, an SNI group, and a PRF group. In the PRF group, L5 dorsal root ganglia received PRF treatment. After paw withdrawal mechanical threshold (PWMT) was examined, the expression levels of CCL2 and nuclear factor kappa B (NF-KB) in spinal dorsal horn were determined.

Results: The PWMT in PRF group increased significantly compared with that of the SNI group $(\mathrm{P}<0.05)$. The CCL2 and NF-kB expression levels in the PRF group were significant lower than those in the SNI group $(P<0.05)$.

Conclusion: NP was effectively alleviated by PRF-mediated reductions in CCL2 expression via inhibition of NF-KB activation in the spinal cord of SNI model rats.

\section{Background}

Neuropathic pain (NP) refers to pain that is started by or induced by a dysfunction or lesion in the somatosensory system ${ }^{1,2}$. The significant morbidity associated with NP represents a challenge to public health, especially for elderly patients ${ }^{3}$. Although many studies have investigated NP development and maintenance mechanisms, the specific details are lacking. Thus, NP remains a clinically refractory pain that requires further research ${ }^{4}$.

Recently, the method of pulsed radiofrequency (PRF) has been used ${ }^{5}$ to treat pain because of its low or absent induction of neurodestruction. PRF is delivered in a pulse of 20 milliseconds ( $\mathrm{ms}$ ) followed by a silent period of $480 \mathrm{~ms}$ to avoid radiofrequency heat lesions ${ }^{6}$. PRF is typically believed to modulate pain pathology and clinical studies have proven its ability to treat several types of NP 7,8 , such as postherpetic neuralgia, painful diabetic neuropathy, and lumbar surgery failure syndrome. In addition, PRF can reduce NP by regulating numerous cytokines, as demonstrated using animal studies ${ }^{9,10}$. However, the mechanism underlying this observation remains unknown.

The chemokine family member chemokine C-C motif ligand 2 (CCL2, also termed monocyte chemoattractant protein 1) plays a key role in the chemotaxis of monocytes to sites of trauma, infection, toxin exposure, and ischemia ${ }^{11}$. CCL2 shows low expression under normal conditions, but is upregulated rapidly in the early stage of nerve injury ${ }^{12}$. The expression levels of CCL2, together with its receptor (CC chemokine family receptor 2 , CCR2), increased in the dorsal horn of the spinal cord in an NP model rat ${ }^{13}$. Interestingly, in non-inflamed rats, CCL2 intrathecal injection resulted obvious nociceptive pain behavior 
14. Moreover, intrathecal preemptive treatment with a CCR2 antagonist markedly reduced nerve injuryinduced pain behavior and inflammatory cytokine release ${ }^{15}$. Thus, there is an implication that CCL2 and CCR2 have critical functions in NP development and maintenance after nerve injury. However, whether PRF reduces NP by modulating CCL2 expression is unknown. The present study aimed to assess the effects of direct PRF application on dorsal root ganglia (DRGs) in the early stage in sciatic nerve injury (SNI) model rats and to determine whether PRF modulates CCL2 expression in the spinal cord.

\section{Materials And Methods}

\section{Experimental animals and groups}

The animal care and use committee of China Medical university approved the experimental procedures (IACUC no. 20190129). Healthy adult Sprague-Dawley (SD) rats weighing 220 to $250 \mathrm{~g}$ were purchased from the Laboratory Animal Center of China Medical University (Shenyang, China). The rats were reared at 22 to $24{ }^{\circ} \mathrm{C}$, with $40-60 \%$ relative humidity, a 12-h light-dark cycle, and ad libitum access to water and food. The rats $(n=60)$ were allocated randomly into three groups: A sham group (exposure of the sciatic nerve with no transection and the RF needle was placed but no energy was delivered), the SNI group (exposure of the sciatic nerve with partial transection and the RF needle was placed but no energy was delivered), and the PRF group (the exposure of the sciatic nerve with partial transection and the RF needle was placed and energy treatment was provided).

\section{Behavioral tests}

The rats were kept in separate metal mesh-bottomed, transparent cages, and allowed to acclimate for $2 \mathrm{~h}$ to test the basal paw withdrawal mechanical threshold (PWMT). The previously described up-down method, using von Frey hairs, was used to assess the PWMT ${ }^{16}$. The first application comprised the $0.4 \mathrm{~g}$ stimulus, which was in the middle of the series. If a positive response (paw licking, shaking, or lifting) occurred within 5 seconds, a thinner filament was applied. In the case of a negative response, a thicker filament was used.

\section{SNI model induction}

Rat SNI surgery was executed following a previously described method ${ }^{17}$. The rats were anesthetized via intraperitoneal injection of $10 \%$ chloral hydrate at $400 \mathrm{mg} / \mathrm{kg}$. Then, an incision was made in the skin of the lateral thigh, followed by blunt dissection of the biceps femoris muscle to reveal the right sciatic nerve together with its three terminal branches (the tibial, common peroneal, and sural nerves). 5-0 silk was used to ligate the tibial and common peroneal nerves tightly. Then, transection of the nerves was performed distal to the ligation, removing a 2-4 mm section of the nerve fiber. Contact or stretching of the intact sural nerve was carefully avoided. The Sham group underwent this procedure without nerve injury. After surgery, the rats were returned to their original cages and environments.

\section{Application of PRF}


In the PRF group, a 21G PRF needle was inserted into the right L5 intervertebral foramen and placed in contact with the surrounding non-neural tissues. RF pluses were generated by the RF instrument (PMG230, Baylis Medical Inc., Montreal, Canada) using the following settings: $2 \mathrm{~Hz}, 42{ }^{\circ} \mathrm{C}$, and $50 \mathrm{~V}$ for 120 seconds. No energy was delivered through the needle in the sham and SNI groups. In a preliminary experiment we found that the peak of PWMT value and CCL2 expression were detected on day 7 after nerve injury; therefore, PRF treatment was administered on day 6.

\section{Specimen collection}

The rats were placed individually in a transparent sealed box for euthanasia. Isoflurane was administered into this box at $5 \mathrm{ml} / \mathrm{kg}$ and the rats were left into the box to breath in the isoflurane until they died (approximately 1-2 $\mathrm{min}$ ). We isolated the $14-6$ spine cord tissues from the rats, which were placed at -80 ${ }^{\circ} \mathrm{C}$ until western blotting analysis.

\section{Western blotting}

The spinal cord of the lumbar (L4-5) ipsilateral to the lesion was collected. Samples were ground in Radioimmunoprecipitation assay (RIPA) on ice. A BCA (bicinchoninic acid) Protein Assay kit (Pierce, Rockford, IL, USA) was used to determine the protein concentration. The samples were subjected to SDSPAGE and then the separated proteins were electroblotted onto a polyvinylidene fluoride membrane.

$5 \%$ Skimmed milk was used to block the proteins on the membrane for $2 \mathrm{~h}$ at room temperature. The membrane was washed using Tris buffered saline Tween (TBST) three times for 5 min each, before being incubated TBST-diluted primary antibodies at $4{ }^{\circ} \mathrm{C}$ overnight. The primary antibodies were anti-PhosphoNF-KB p65(Ser536) antibody (1:1000, 3303; Cell Signaling Technology, Danvers, MA, USA), anti-CCL2 antibody (1:2000, ab25124; Abcam, Cambridge, MA, USA), and anti- $\beta$-actin (1:4000, 20536-1-AP, Proteintech, Rosemont, IL, USA). The membranes were then washed in TBST ( $5 \mathrm{~min}$, three times) and incubated with horseradish peroxidase (HRP)-conjugated goat anti-rabbit secondary antibodies (1:10000, SA00001-2, Proteintech) for $2 \mathrm{~h}$ at room temperature. The membranes were washed again in TBST (10 min, three times), and then the immunoreactive proteins were visualized using electrochemiluminescence (ECL) reagents, and results were analyzed using the Gel Doc XR and ChemiDoc XRS system (Biorad, Hercules, CA, USA). The levels of the immunoreactive proteins were assessed quantitively using the grayscale values.

\section{Statistical analysis}

All statistical analyses were accomplished using SPSS 18.0 (IBM Corp., Armonk, NY, USA). All date are shown as the mean \pm standard error. Repeated-measures analysis of variance (ANOVA) was used to analyze the PWMT. One way ANOVA was used to analyze the levels of CCL2 and phospho-NF-KB p65. Statistical significance was accepted at $P<0.05$ was considered significant.

\section{Results}




\section{Changes in behavior}

Among the three groups, there were no significant difference in the PWMT before transection of the sciatic nerve. There were significant decreases in the PWMT score on day 1 (5.551 \pm 0.934 vs. $0.795 \pm$ $0.316), 3(9.645 \pm 1.686$ vs. $0.319 \pm 0.134), 7$ (18.657 \pm 2.788 vs. $0.240 \pm 0.151)$, and 10 (17.904 \pm 2.662 vs. $1.131 \pm 0.454)$ after nerve injury in the SNI group compared with that in the sham group $(\mathrm{P}<0.001)$. There were also significant decreases in the PWMT score on day 1 (5.551 \pm 0.934 vs. $0.840 \pm 0.332), 3$ (9.645 \pm 1.686 vs. $0.353 \pm 0.1352), 7(18.657 \pm 2.788 v s .2 .925 \pm 0.754)$, and 10 (17.904 $\pm 2.662 v s$. $6.923 \pm 1.228)$ after nerve injury in the PRF group compared with that in the sham group $(P<0.01)$. No significant difference in the PWMT score was observed between the SNI and PRF group on day 0,1 , and 3 days in the absence of PRF treatment after sciatic nerve partial transection. After PRF application, there were significant increases in the PWMT score on day $7(0.240 \pm 0.151$ vs. $2.925 \pm 0.754)$ and $10(1.131 \pm$ 0.454 vs. $6.923 \pm 1.228)$ in the PRF group compared with that in the SNI group $(P<0.05)(F i g .1)$.

\section{Western blotting analysis of CCL2 expression}

After sciatic nerve section injury, the level of CCL2 increased immediately on day 1,3, 7 and 10. The peak expression in the SNI group occurred at 7 days after nerve injury and was 2.46 times higher than that in the sham group $(\mathrm{P}<0.05)$ (Fig. 2a). After PRF treatment, the level of CCL2 declined significantly in the PRF group compared with that in the SNI group. The relative protein level of CCL2 in the PRF group was $40.4 \%$ lower compared with that in the SNI group on day 7 (1 day after PRF) and was $66.2 \%$ lower than that in the SNI group on day 10 (4 days after PRF) ( $P<0.05$, Fig. $2 b)$.

\section{Western blotting analysis of NF-KB expression}

After sciatic nerve section injury, the level of NF-KB increased significantly on days 3,7 , and 10 . The peak expression in the SNI group occurred on day 7 after nerve injury and was 1.79 times higher than that observed in the sham group $(\mathrm{P}<0.05)$ (Fig. 3a). After PRF treatment, the NF-KB levels decreased significantly declined in the PRF group. The relative protein expression of NF-KB in the PRF group was $25.6 \%$ lower than that in the SNI group on day 7 (1 day after PRF) and was $19.3 \%$ lower compared with that in the SNI group on day 10 (4 days after PRF) ( $P<0.05$, Fig. 3b).

\section{Discussion}

\section{PRF alleviates hyperalgesia in SNI rats}

In the present study, we demonstrated that after partial sciatic nerve transection, the PWMT values in the SNI group decreased significantly compared with those in the sham group. The SNI model can reliably simulate the clinical manifestations of NP in humans, for example hyperpathia, allodynia, and spontaneous pain ${ }^{18}$. There was a linear decline in the PWMT value after nerve injury, comprising a rapid decline from the first day, reaching a minimum at 7 days, before rising slowly. Our findings are consistent with those of a previous study ${ }^{19}$ and indicated that the NP model was established successfully. 
The most severe pain was observed at 7 days after partial nerve transection; therefore, PRF was applied to the DRG at 6 days after nerve injury. After 1 day of PRF treatment, there was a significant improvement in mechanical allodynia, which further improved after another 4 days. These results were similar to those of previous studies ${ }^{20,21}$, which confirmed that PRF treatment of the DRG could alleviate hyperalgesia and allodynia significantly in an NP model.

The DRG is found at the upper region of the intervertebral foramen, which contains the primary class of sensory neurons and glial cells. After nerve injury, the activated neurons and glial cells in the DRG release many kinds of inflammatory cytokines, which are involved in nociceptive transmission and central sensitization. The DRG has an important role in NP development and maintenance, making it an excellent clinical target for chronic pain treatment ${ }^{22,23}$. Therefore, in the present study, the PRF current duration was applied on the L4-5 DRG to achieve excellent pain relief.

\section{CCL2 and NF-KB levels increased in the SNI model}

CCL2 is constitutively expressed in neurons and glial cells, and is dramatically upregulated in the early stage of peripheral nerve injury 24,25 . Nuclear factor kappa B (NF-KB) is a key transcription factor that regulates the expression of many genes (e.g., those encoding cytokines, chemokines, and neural nitric oxide synthase (nNOS)) that are important in NP development ${ }^{26,27}$. After being phosphorylated and activated in response to cell stimulation, NF-kB acts as a critical element in CCL2 induction ${ }^{28}$. In the present study, we found that CCL2 and NF-KB levels in the spinal cord increased immediately after sciatic nerve partial transection. The levels of CCL2 and NF-KB both peaked at 7 days after nerve injury and then gradually declined, which indicated that CCL2 expression might be regulated primarily by NF-KB. These results were in agreement with the results of $\mathrm{GaO}^{29}$, who found that the increase in CCL2 expression could be observed from days 1 to 10 , and peaked at day 7 in the spinal cord in an SNI model.

\section{PRF might exert an analgesic function by reducing CCL2 levels in the spinal cord}

Western blotting analysis revealed that the CCL2 contents in the s pinal cord of the PRF group decreased significantly on day 7 and 10 compared with those in the SNI group. The trend of NF-KB levels was the same as that for CCL2 in the SNI and PRF groups. NF-KB is also a critical upstream regulator of CCL2 expression. These results suggested that PRF reduced CCL2 levels by inhibiting the phosphorylation and activation of NF-KB in the rat spinal cord. CCL2 has a low expression levels in neurons and glial cells under normal conditions. Once peripheral nerve injury occurs, CCL2 could be released immediately, which would lead to glial cell activation, ultimately causing the release of several various pain regulators, including nerve growth factor, interleukin-1 $\beta$ (IL-1 $\beta$ ), tumor necrosis factor- $a$ (TNF- $\alpha$ ), and CCL2 ${ }^{30,31}$. These pain regulators are involved in central sensitization and lead to the development and maintenance of $\mathrm{NP}^{32}$. According to our experimental results, we hypothesized that PRF inhibits this chain reaction by downregulating the expression of CCL2, which might represent one of the mechanisms by which PRF treatment achieves analgesia. 


\section{Limitations}

The results or the present study demonstrated that PRF could reduce hyperalgesia and decrease CCL2 and NF-KB level in the spinal cord in the early stage of NP in the SNI model; however, the detailed relationships among hyperalgesia, CCL2, and PRF require further research. We observed the changes of pain threshold, and CCL2 and NF-KB levels only up to 4 days after PRF administration; thus, to determine the long-term efficacy of PRF, longer follow-up is required. We only examined the expression of CCL2 in the spinal cord, but not in other parts, including the DRG and the dorsal horn of the spinal cord. Whether PRF has same treatment effect in the presence of antagonists of CCL2 or CCR2 also needs further experimental clarification.

\section{Conclusions}

PRF effectively alleviated hyperalgesia after administrated to dorsal root ganglion in early stage after nerve injury. The mechanism might reduce the expression of the CCL2 though inhibiting NF-KB activation in the spinal dorsal horns, which would then prevent the transmission of pain signals to the CNS.

\section{Declarations}

\section{Funding statement}

This study was financially supported by a grant from Liaoning Education Department Foundation (No. QNZR2020007). The sponsor of the study did not participate in data collection, data analysis, data interpretation, or the writing of the manuscript.

\section{Acknowledgements}

We would like to thank all the students, doctors and technicians involved in the current study.

\section{Authors' contributions}

Cheng-fu Wan performed the majority of the experiments, designed the study and acquired data, was involved in the analysis and interpretation of data, participated in drafting the manuscript, in critical revision of important content and gave final approval of the version to be released.

Bo-han Zhang and Dao-song Dong contributed to data interpretation and analysis. Tao Song made a substantial contribution to the experimental design, drafting and critically revising the manuscript. all of the authors have read and approved the final manuscript.

\section{Ethics approval and consent to participate}

all experiments were conducted in accordance with the Guiding Principles for research involving animal and Human Beings, and the experimental procedures were approved by the animal care and use 
committee of china Medical university (iacuc no. 20190129).

\section{Patient consent for publication}

not applicable.

\section{Competing interests}

All the authors declare that they have no competing interests.

\section{References}

1. Treede RD JT, Campbell JN, Cruccu G, Dostrovsky JO, Griffin JW, et al. Neuropathic pain: redefinition and a grading system for clinical and research purposes. Neurology. 2008;70:1630-5.

2. Miao Fu LM, Hao Ren, Fang Luo. Pulsed radiofrequency inhibits expression of P2 $\times 3$ receptors and alleviates neuropathic pain induced by chronic constriction injury in rats. Chin Med J. 2019;132(14):1706-12.

3. Campbell JN MR. Mechanisms of neuropathic pain. Neuron. 2006;52:77-92.

4. Mercer SJ CS, Tong JL, Connor DJ, de Mello WF. The early detection and management of neuropathic pain following combat injury. J R Army Med Corps. 2009;155:94 - 8.

5. Wan C DD, Song T. High-Voltage, Long-Duration Pulsed Radiofrequency on Gasserian Ganglion Improves Acute/Subacute Zoster-Related Trigeminal Neuralgia: A Randomized, Double-Blinded, Controlled Trial. Pain physician. 2019;22(4):361-8.

6. SluijterME vM. Pulsed radiofrequency. Pain Med. 2007;8:388-389. author reply 390-1.

7. Shi Y WW. Treatment of neuropathic pain using pulsed radiofrequency: a meta-analysis. Pain Physician. 2016;19:429 - 44.

8. Shanthanna H CP, McChesney J, Thabane L, Paul J. Pulsed radiofrequency treatment of the lumbar dorsal root ganglion in patients with chronic lumbar radicular pain: A randomized, placebocontrolled pilot study. J Pain Res. 2014;7:47-55.

9. Lee JB BJ, Choi IS, Kim Y, Lee JS. The effect of pulsed radiofrequency applied to the peripheral nerve in chronic constriction injury rat model. Ann Rehabil Med. 2015;39:667-675.

10. Yeh CC WZ, Chen JC, Wong CS, Huang CJ, Wang JS, et al. Association between extracellular signalregulated kinase expression and the anti-allodynic effect in rats with spared nerve injury by applying immediate pulsed radiofrequency. BMC Anesthesiol. 2015;15:92.

11. Calvo M BD. The mechanisms of microgliosis and pain following peripheral nerve injury. Exp Neurol 2012;234:271 - 82.

12. Thacker MA CA, Bishop T, Grist J, Yip PK, Moon LD, Thompson SW, et al. CCL2 is a key mediator of microglia activation in neuropathic pain states. Eur J Pain. 2009;13:263 - 72. 
13. White FA SJ, Waters SM, Ma C, Ren D, Ripsch M, Steflik J, et al. Excitatory monocyte chemoattractant protein-1 signaling is up-regulated in sensory neurons after chronic compression of the dorsal root ganglion. Proc Natl Acad Sci. 2005;102:14092-7.

14. Dansereau MA GR, Pohl M, Pommier B, Mechighel P, Mauborgne A, et al. Spinal CCL2 pronociceptive action is no longer effective in CCR2 receptor antagonist-treated rats. J. Neurochem. 2008;106:757 69.

15. Kwiatkowski K PA, Rojewska E, Makuch W, Mika, J. The RS504393 influences the level of nociceptive factors and enhances opioid analgesic potency in neuropathic rats. J. Neuroimmune Pharmacol. 2017;12:402 - 19.

16. Chaplan SR BF, Pogrel JW, Chung JM, Yaksh TL. Quantitative assessment of tactile allodynia in the rat paw. J Neurosci Methods. 1994;53(1):55-63.

17. Liu Z LY, Wang H, Lu Z, Chen J, Huang Q, et al. LncRNA expression in the spinal cord modulated by minocycline in a mouse model of spared nerve injury. J Pain Res. 2017;10:2503-14.

18. Decosterd I WC. Spared nerve injury: an animal model of persistent peripheral neuropathic pain. Pain. 2008;87(2):149 - 58.

19. Richner M BO, Nykjaer A, Vaegter CB. The spared nerve injury (SNI) model of induced mechanical allodynia in mice. $J$ Vis Exp. 2011;54:3092.

20. Ren-Yu Huang C-CL, Shih-Ying Tsai, Chen-Tung Yen, Chii-Wann Lin, Tsung-Chi Chen, et al. Rapid and Delayed Effects of Pulsed Radiofrequency on Neuropathic Pain: Electrophysiological, Molecular, and Behavioral Evidence Supporting Long-Term Depression. Pain Physician. 2017;20:E269-83.

21. Zihao Liu YL, Honghua Wang, Zhenhe Lu, Jinsheng Chen, Qiaodong Huang, et al. LncRNA expression in the spinal cord modulated by minocycline in a mouse model of spared nerve injury. Journal of Pain Research. 2017;10:2503-14.

22. Berta T QY, Tan PH, Ji RR. Targeting dorsal root ganglia and primary sensory neurons for the treatment of chronic pain. Expert Opin Ther Targets. 2017;21(7):695-703.

23. ES K. The dorsal root ganglion in chronic pain and as a target for neuromodulation: A review. Neuromodulation. 2015;18:24-32.

24. Jung H TP, White FA, Miller RJ. Monocyte chemoattractant protein-1 functions as a neuromodulator in dorsal root ganglia neurons. J Neurochem. 2008;104(1):254 - 63.

25. Zhang ZJ JB, Gao YJ. Chemokines in neuron-glial cell interaction and pathogenesis of neuropathic pain. Cell Mol Life Sci. 2017;74(18):3275-91.

26. Eugene SF ZY, Jacqueline Sagen, Candiotti KA, Morton PD, Liebl DJ, et al. Transgenic Inhibition of Glial NF-kappa B Reduces Pain Behavior and Inflammation after Peripheral Nerve Injury. Pain. 2010;148(3):509 - 18.

27. Charo IF RR. The many roles of chemokines and chemokine receptors in inflammation. $N$ Engl $J$ Med. 2006;354(6):610 - 21. 
28. Ogura N SK, Akutsu M, Tobe M, Kuyama K, Kuboyama N,et al. MCP-1 production in temporomandibular joint inflammation. J Dent Res. 2010;89(10):1117-22.

29. Gao YJ ZL, Samad OA, Suter MR, Yasuhiko K, Xu ZZ, et al. JNK-induced MCP-1 production in spinal cord astrocytes contributes to central sensitization and neuropathic pain. $J$ Neurosci. 2009;29(13):4096 - 108.

30. AM. Illias AG, HJ Zhang, AK Kosturakisa, PM Dougherty. Chemokine CCL2 and its receptor CCR2 in the dorsal root ganglion contribute to oxaliplatininduced mechanical hypersensitivity. Pain. 2018;159:1308-16.

31. Zhang ZJ JB, Gao YJ. Chemokines in neuron-glial cell interaction and pathogenesis of neuropathic pain. Cell Mol Life Sci. 2017;74:3275-91.

32. Kwiatkowski K PA, Rojewska E, Makuch W, Mika, J. Epigenetic regulation of CCchemokine ligand 2 in nonresolving inflammation. Biomol Concepts. 2014;5:265 - 73.

\section{Figures}

\section{\% sham $\quad \mathbf{S N I} \quad$ P PRF}

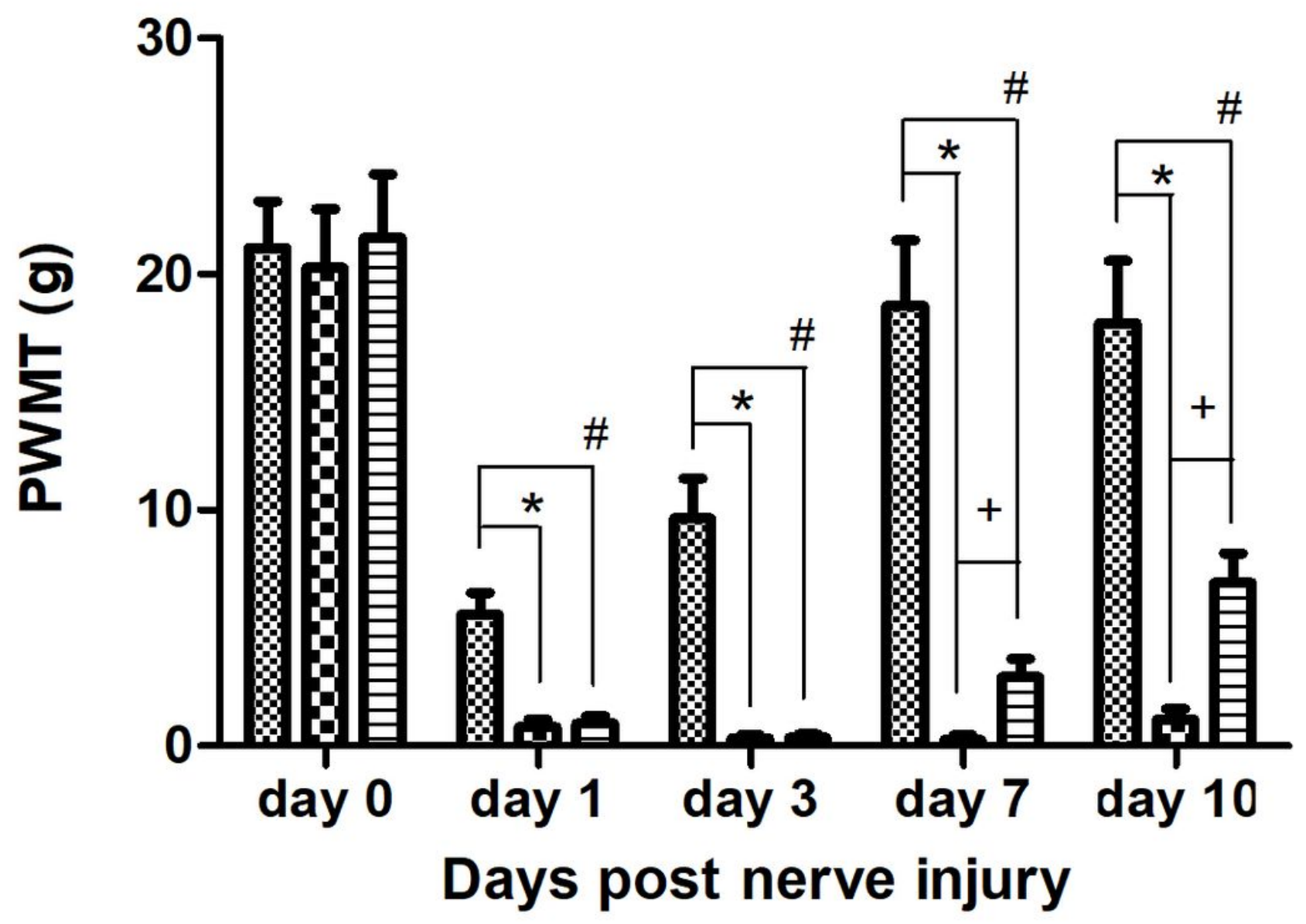

Figure 1 
The change of PWMT after sciatic nerve section injury; $* \mathrm{P}<0.001$, sham group vs. SNI group; $\# \mathrm{P}<0.01$, sham group vs. PRF group; $+P<0.05$, SNI group vs. PRF group. Data are expressed as the mean \pm standard error.

\section{$\otimes$ sham $\mathrm{ESNI} \equiv \mathrm{PRF}$}

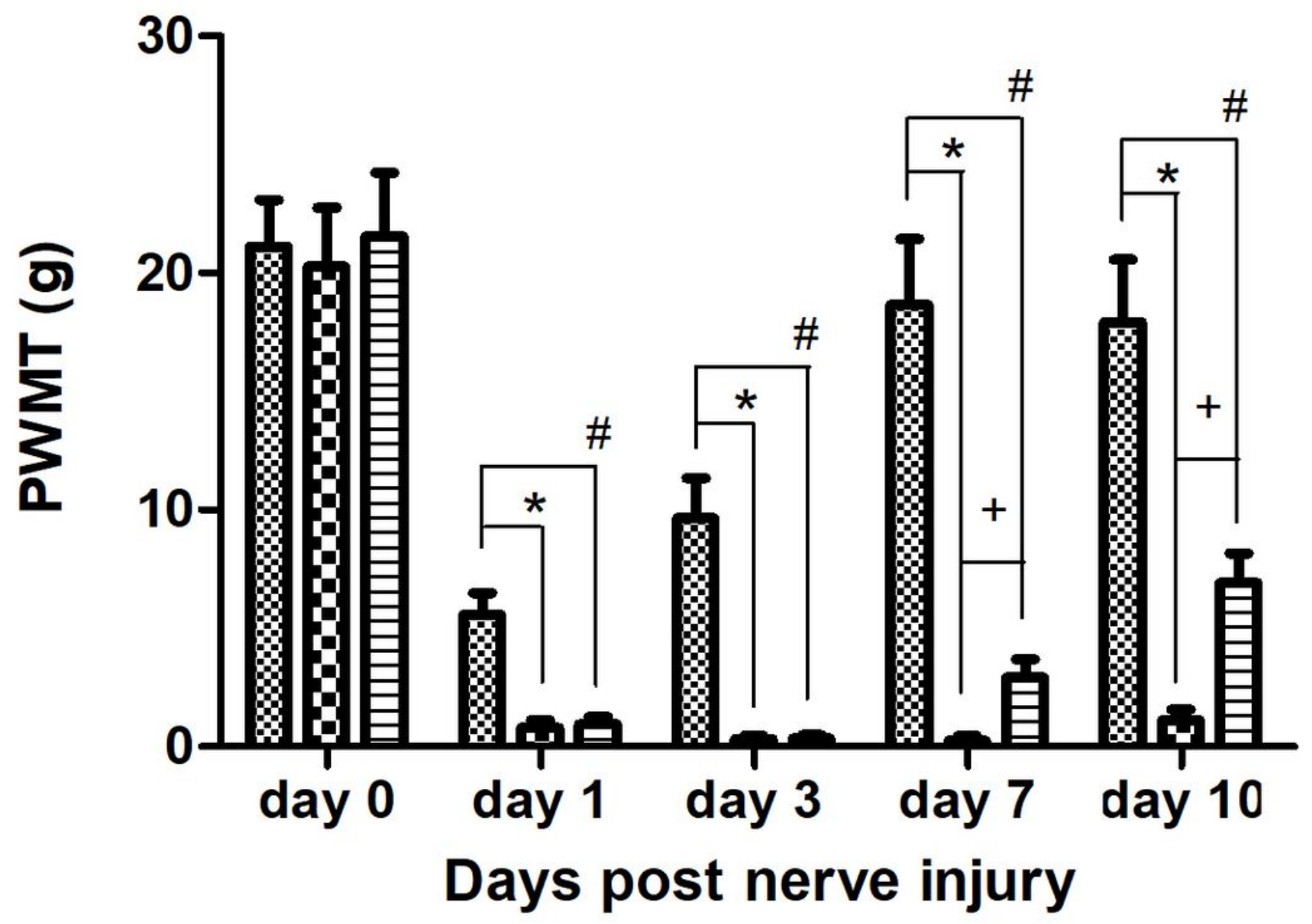

Figure 1

The change of PWMT after sciatic nerve section injury; $* \mathrm{P}<0.001$, sham group vs. SNI group; $\# \mathrm{P}<0.01$, sham group vs. PRF group; $+P<0.05$, SNI group vs. PRF group. Data are expressed as the mean \pm standard error. 


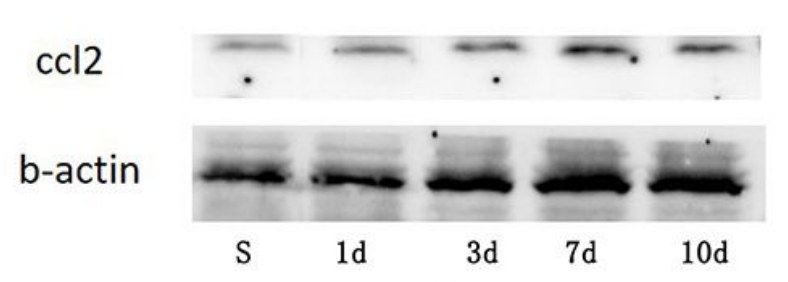

Relative CCL-2 expression

sham $\mathrm{BNN}$

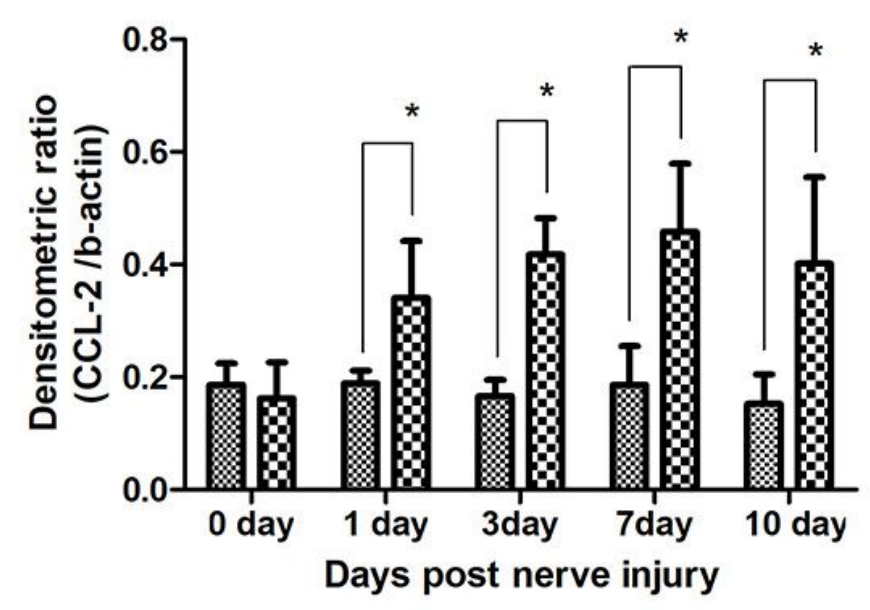

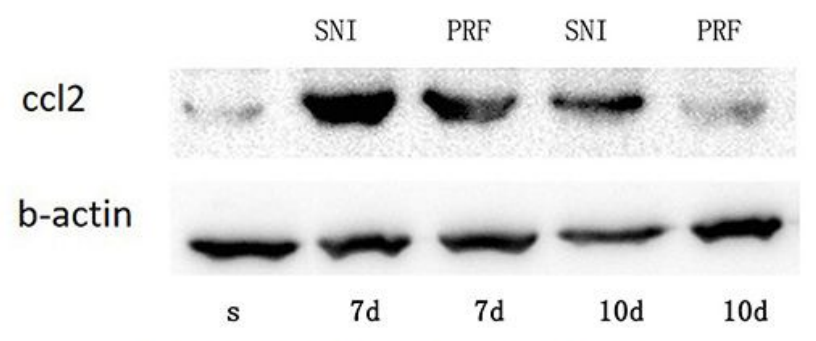

Relative CCL-2 expression

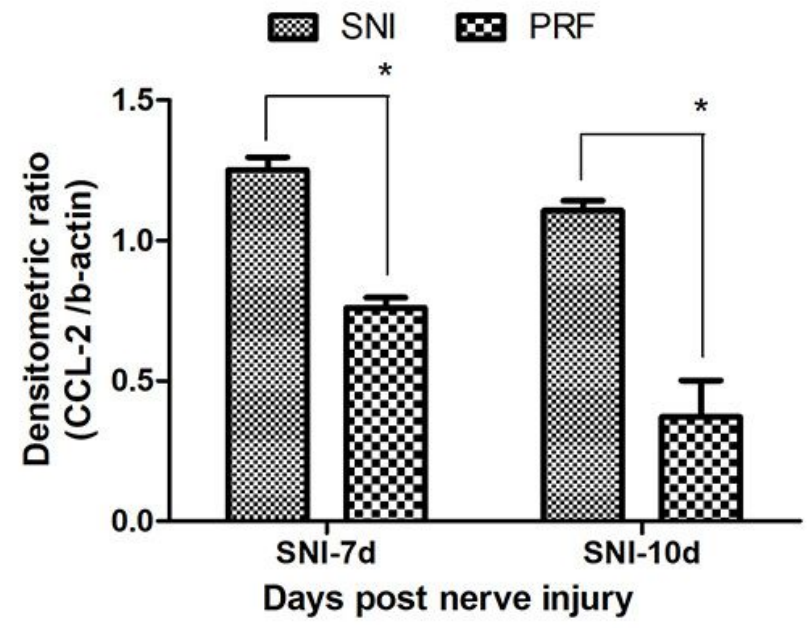

Figure 2

2a: The relative protein expression of CCL2 in spinal cord after sciatic nerve section injury. $* \mathrm{P}<0.05$, sham group vs. SNI group. Data are expressed as the mean \pm standard error. $2 \mathrm{~b}$ : The relative protein expression of CCL2 in the spinal cord on 7 and 10 days after sciatic nerve section injury. $* \mathrm{P}<0.05, \mathrm{SNI}$ group vs. PRF group. Data are expressed as the mean \pm standard error. 


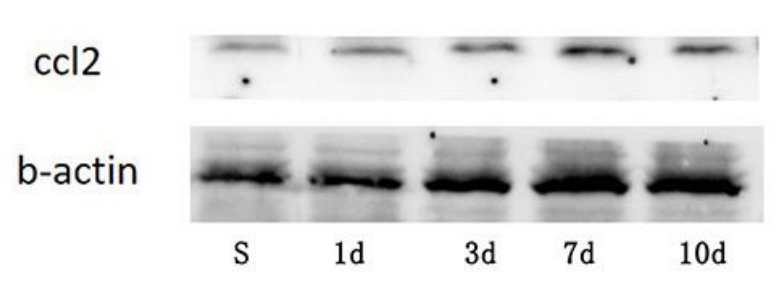

Relative CCL-2 expression

m sham $\mathrm{SNI}$

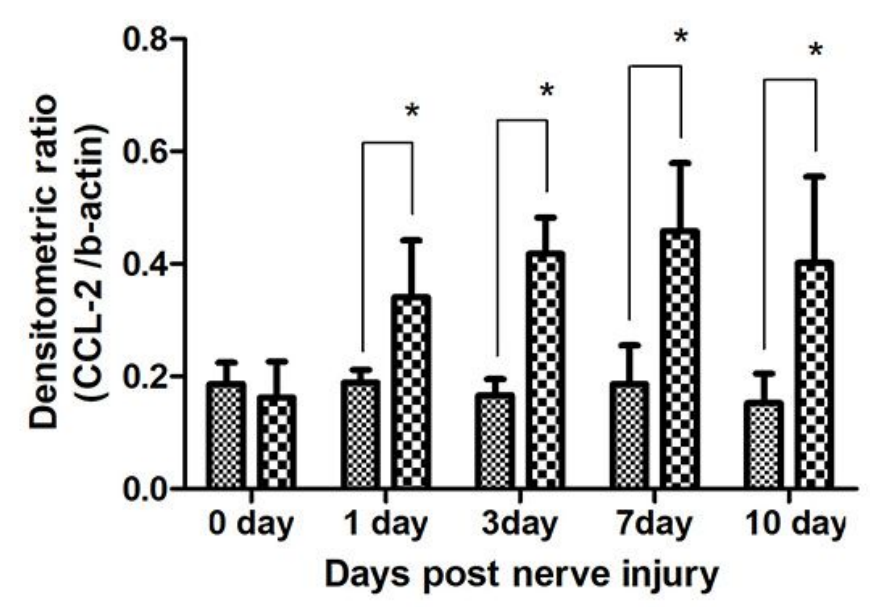

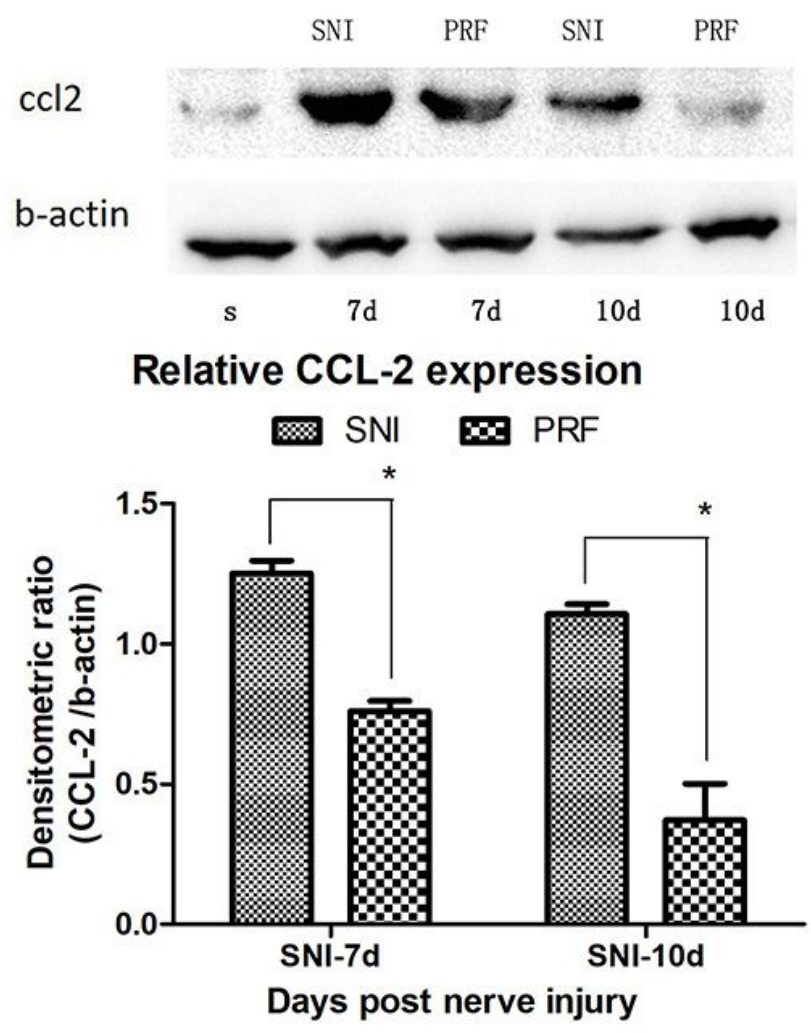

Figure 2

2a: The relative protein expression of CCL2 in spinal cord after sciatic nerve section injury. $* \mathrm{P}<0.05$, sham group vs. SNI group. Data are expressed as the mean \pm standard error. $2 \mathrm{~b}$ : The relative protein expression of CCL2 in the spinal cord on 7 and 10 days after sciatic nerve section injury. $* \mathrm{P}<0.05, \mathrm{SNI}$ group vs. PRF group. Data are expressed as the mean \pm standard error. 

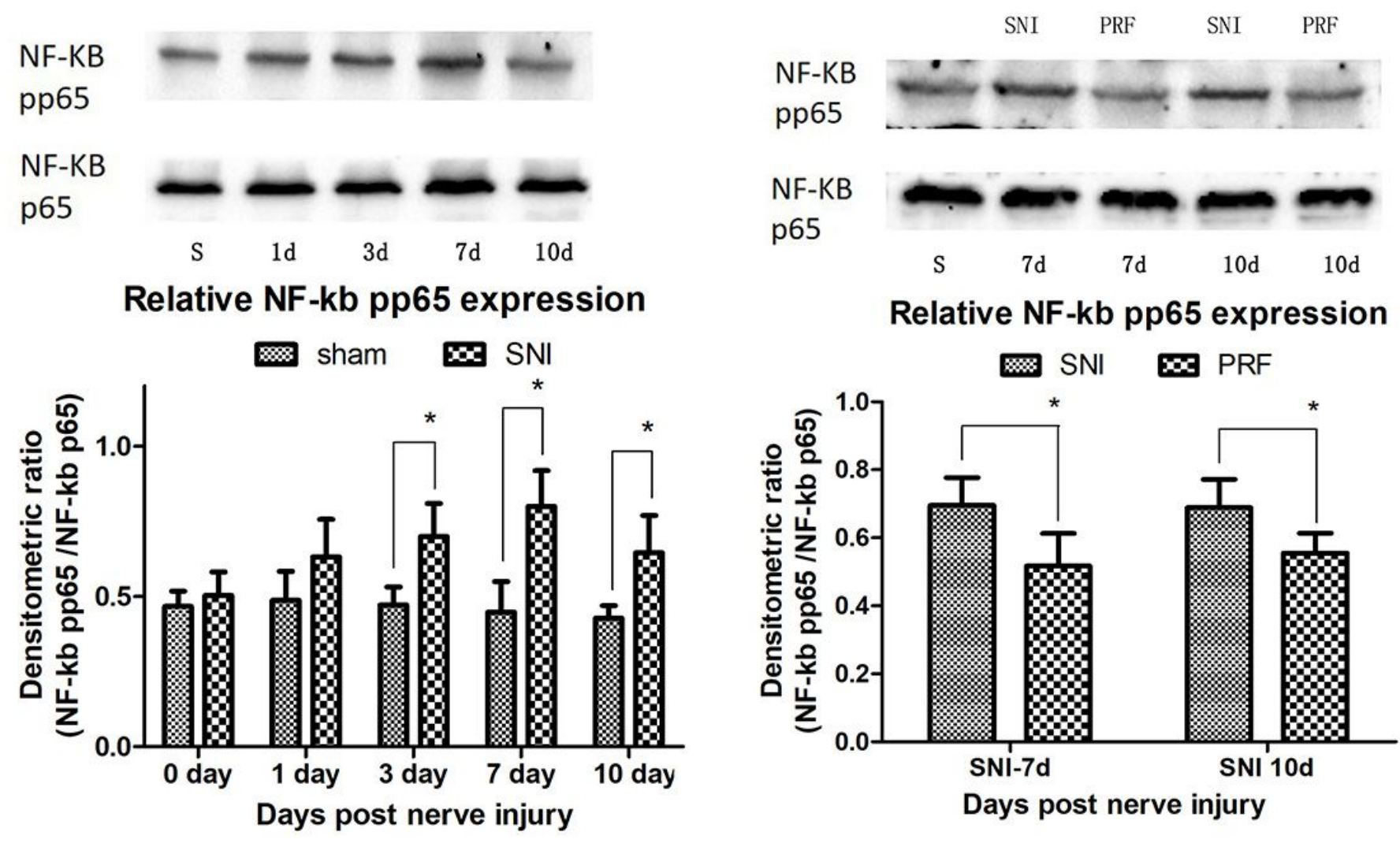

Relative NF-kb pp65 expression

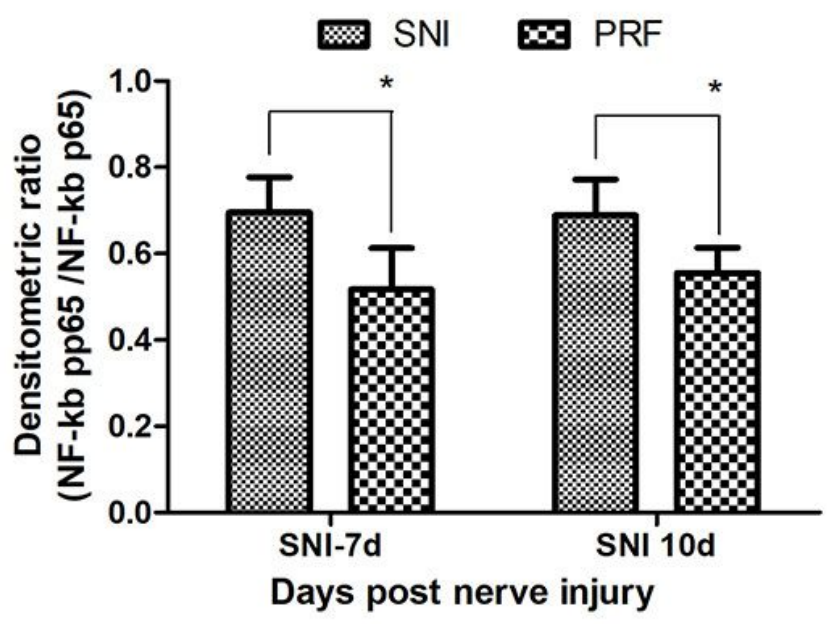

Figure 3

3a: The relative protein expression of NF-KB in spinal cord after sciatic nerve section injury. $* P<0.05$, sham group vs. SNI group. Data are expressed as the mean \pm standard error. $3 \mathrm{~b}$ : The relative protein expression of NF-kB in spinal cord on 7 and 10 days after PRF. $* \mathrm{P}<0.05$, SNI group vs. PRF group. Data are expressed as the mean \pm standard error. 

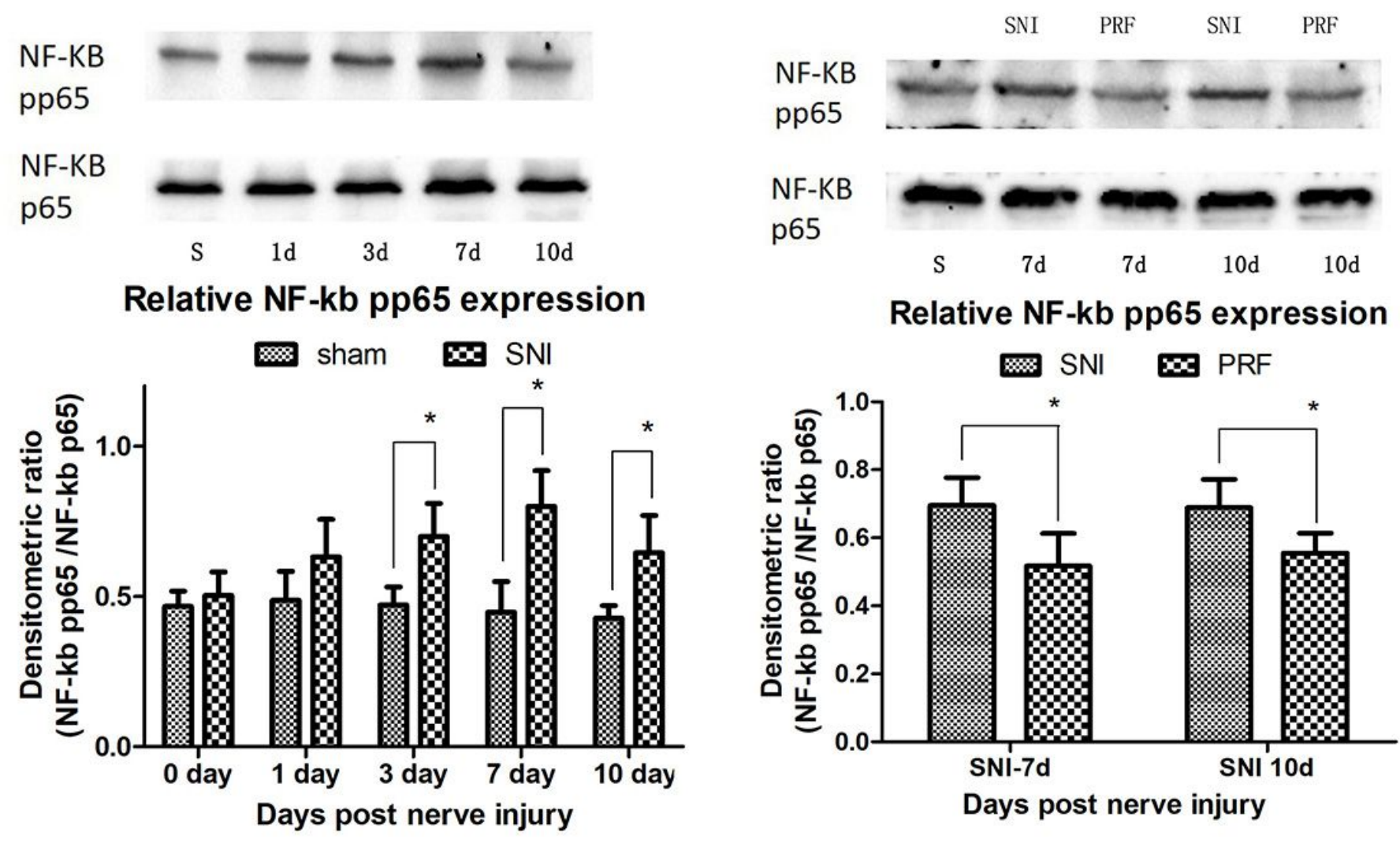

Relative NF-kb pp65 expression

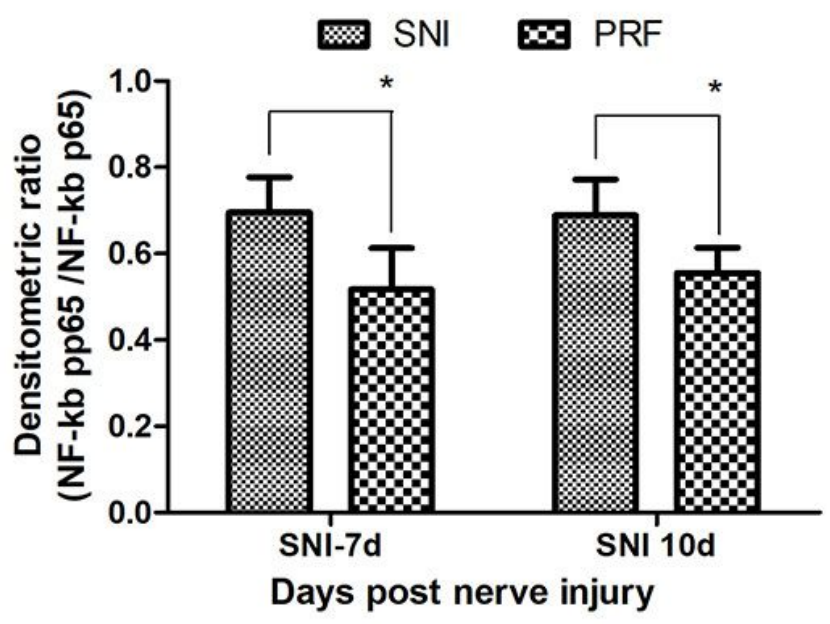

Figure 3

3a: The relative protein expression of NF-KB in spinal cord after sciatic nerve section injury. $* P<0.05$, sham group vs. SNI group. Data are expressed as the mean \pm standard error. $3 \mathrm{~b}$ : The relative protein expression of NF-kB in spinal cord on 7 and 10 days after PRF. $* \mathrm{P}<0.05$, SNI group vs. PRF group. Data are expressed as the mean \pm standard error. 